\title{
Chemical composition of surface and structure of defects in diamond single crystals produced from detonation nanodiamonds
}

\author{
S. V. Kidalov ${ }^{1}$, V. V. Shnitov ${ }^{1}$, M. V. Baidakova ${ }^{1}$, M. Brzhezinskaya ${ }^{2}$, A. T. Dideikin ${ }^{1}$, M. S. Shestakov ${ }^{1}$, \\ D. A. Smirnov ${ }^{2}$, I. T. Serenkov ${ }^{1}$, V. I. Sakharov ${ }^{1}$, V. V. Sokolov ${ }^{1}$, N. I. Tatarnikov ${ }^{1}$, A. Ya. Vul ${ }^{1}$ \\ ${ }^{1}$ Ioffe Institute, Polytechnicheskaya 26, St. Petersburg, 194021, Russia \\ ${ }^{2}$ Helmholtz Zentrum Berlin Mat \& Energie, D-12489 Berlin, Germany \\ Kidalov@mail.ioffe.ru
}

PACS 78.55.-m, 78.70.-g

DOI 10.17586/2220-8054-2018-9-1-21-24

\begin{abstract}
We present the results from our investigation of the structure and composition of microcrystalline diamonds obtained by sintering at high pressures and at high temperatures of detonation nanodiamond particles. Using XPS, XAS and photoluminescence spectroscopy, we found that the surface's chemical composition and a defects structure of microcrystalline diamonds significantly differ from the initial detonation nanodiamonds. This indicates the essential transformation of structure and composition of initial detonation nanodiamonds particles during the formation of single crystals at high pressure and temperature.
\end{abstract}

Keywords: detonation nanodiamond, HPHT sintering, single crystal, XPS and XAS spectroscopy, photoluminescence, defects.

Received: 20 June 2017

Revised: 27 September 2017

\section{Introduction}

The possibility of growing bulk single crystals from detonation nanodiamond (DND) particles by oriented attachment in the presence of alcohols or/and hydrocarbons was predicted and experimentally demonstrated by our group in previous works [1-3]. It is assumed that diamond single crystals of size between $500 \mathrm{~nm}$ and $15 \mathrm{mcm}$ are formed directly from $4-5 \mathrm{~nm}$ sized diamond nanoparticles under conditions of high pressure and high temperature $(\mathrm{HPHT})\left(P \approx 7 \mathrm{GPa}, T \approx 1300-1700{ }^{\circ} \mathrm{C}\right)$. One of the most promising applications of synthesized diamond microcrystals is related to the formation of luminescent impurity centers inside diamond lattice from their own system of defects originating during crystal growth. The distinction of composition and structure of lattices defects in sintered microcrystals and initial DND particles was found for the first time by ESR [4,5]. The goal of present work is studying transformation of composition and structure of defects of DND resulting from forming microcrystals by HPHT sintering, as well as estimating the possibility of obtaining of the luminescense centers (nitrogen-vacancy) in microcrystals obtained by sintering of DND particles.

\section{Experimental details}

Samples of highly purified deagglomerated DND were obtained in accordance with the procedure described in [6,7]. Samples of diamond single crystals of size between $500 \mathrm{~nm}$ and $2 \mu \mathrm{m}$ were prepared by HPHT sintering of DND at the pressure of $\sim 7 \mathrm{GPa}$ and the temperature of $\sim 1550{ }^{\circ} \mathrm{C}$ for $8-15 \mathrm{~s}$ in the presence of hydrocarbons (hexane). Initial DND particles for spectral measurements were deposited to the surface of silicon substrates by aerosolization. The thickness of layer was about $100 \mathrm{~nm}$. Microcrystals obtained by sintering of DND, were dented into the surface of copper substrates. The thicknesses of obtained layers were approximately $50 \mu \mathrm{m}$.

For transformation of the structure of defects in the initial DND and in the synthesized diamond microcrystals, we applied ion irradiation and annealing. We used ions of nitrogen $\left(\mathrm{N}^{+}\right)$with energy $E=227 \mathrm{keV}$ and doze $D=2.5 \cdot 10^{15} \mathrm{~cm}^{-2}$. The annealing was carried out at temperature $T=750{ }^{\circ} \mathrm{C}$ in a vacuum chamber $\left(P=10^{-8}\right.$ Torr $)$ for $1 \mathrm{~h}$.

X-ray photoelectron (XPS) and CK edge X-ray absorption (XAS) spectra were measured at the RussianGerman beamline of electron storage ring BESSY-II (Helmholtz-Zentrum Berlin) using the beam line ultra-high vacuum (UHV) experimental station [8]. The XPS spectra were acquired at fixed photon energies using hemispherical analyzer SPECS Phoibos 150 operated in constant-pass-energy mode. The CK edge XAS spectra were measured in the mode of total electron yield (TEY) by recording a value of the sample drain current as a function of the energy of the incident photons.

Photoluminescence (PL) studies were performed at room temperature using spectrometer Ocean Optics Maya2000 Pro with spectral range $200-1100 \mathrm{~nm}$ and resolution $0.45 \mathrm{~nm}$. The excitation wavelength was $532 \mathrm{~nm}$. 


\section{Results and discussion}

Typical SEM image of microdiamond particles produced by HPHT sintering of DNDs is presented in Fig. 1a. Respective survey XPS spectra of initial DND and synthesized diamond microcrystals are presented in Fig. $1 \mathrm{~b}$.

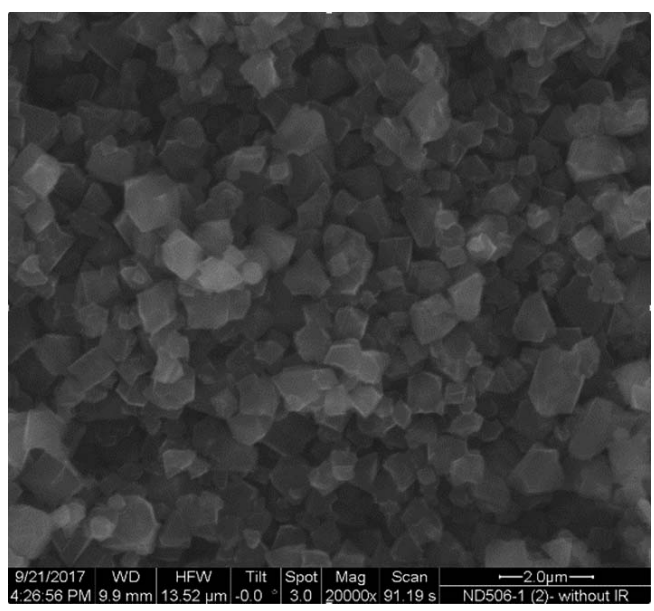

a

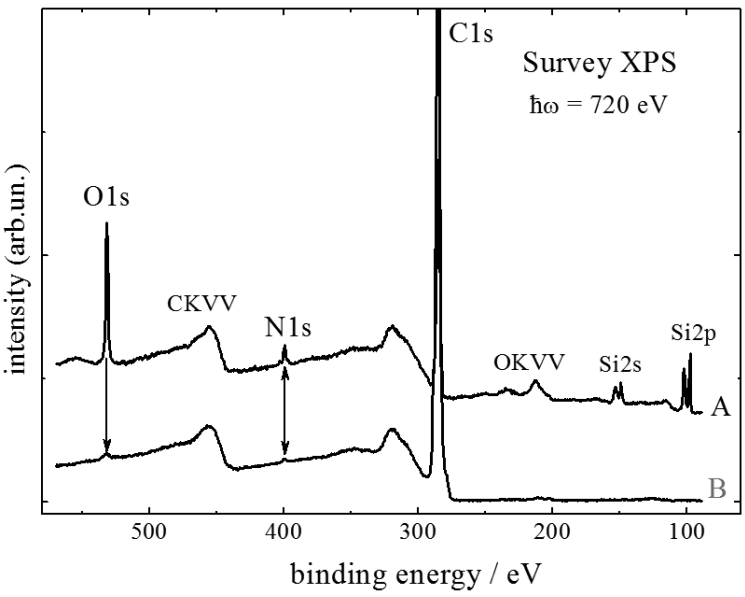

b

FIG. 1. a) SEM images of synthesized diamond microcrystals obtained by sintering of DND under HPHT conditions. Scale ruler $-2 \mu \mathrm{m}$. The average crystal size is $1 \mu \mathrm{m}$. b) Survey XPS spectra of DND (curve A) and of synthesized diamond microcrystals (curve B) recorded at the energy of monochromatic synchrotron radiation equals to $720 \mathrm{eV}$

Figure 1a, confirms the above-mentioned size range for synthesized diamond microcrystals and gives proof for their single crystal nature. Fig. 1b expectedly shows that initial DND and synthesized diamond microcrystals have almost the same chemical composition, which includes three elements: carbon, oxygen and nitrogen. However, the obtained XPS spectra clearly show that unlike initial DNDs, the chemical composition of synthesized diamond microcrystals does not contain any discernible traces of nitrogen $(\mathrm{N} 1 \mathrm{~s}$ core level peak $(\mathrm{BE} \sim 401 \mathrm{eV})$ is completely absent in the XPS spectra of synthesized diamond microcrystals). Thus, we may assume that process of HPHT sintering of DND is accompanied by stringent removal of nitrogen from the near surface layers of the produced diamond single crystals.

Figure 2 presents CK edge XAS spectra of the same diamond particles measured, respectively, on the untreated samples (spectra 1), on the samples first irradiated by nitrogen ions with the energy of $227 \mathrm{keV}$ and the dose of $2.6 \cdot 10^{15}$ ions $/ \mathrm{cm}^{2}$ (spectra 2) and then on the same samples annealed during $1 \mathrm{~h}$ at the temperature of $750{ }^{\circ} \mathrm{C}$ under high vacuum (spectra 3 ).

Analysis of XAS spectra clearly shows that besides removal of nitrogen, DND sintering is also accompanied by essential transformation of their bulk electronic structure and of their surface chemistry. Proof for this is shown by a number of new spectral features which were absent in the XAS of initial DNDs (spectrum 1A), but they appear in respective spectra (spectrum 1B) of synthesized diamond microcrystals. These are the following post-edge $(h \omega>289 \mathrm{eV})$ features: a very narrow $(\sim 0.3 \mathrm{eV})$ peak of $\mathrm{C} 1$ s core exciton at $\sim 289.3 \mathrm{eV}$, broad bulk $\sigma^{*}$-resonances at $\sim 290.8, \sim 295.3$ and distinctive shoulder at $\sim 303.3 \mathrm{eV}$. All of these features are commonly considered signs of high structural perfection of diamond $[9,10]$ and point that HPHT sintering converts DNDs into single crystal diamonds whose bulk electronic structure and degree of structural perfection are very similar to quality synthetic diamonds.

The prominent distinction in the shape of pre-edge regions $(h \omega<289 \mathrm{eV})$ points out the difference in the surface chemistry of initial DNDs and synthesized diamond microcrystals. It could be related to the strong functionalization of synthesized diamond microcrystals surface by $\mathrm{C}-\mathrm{H}$ groups revealing itself in high intensity of $\sigma^{*}(\mathrm{C}-\mathrm{H})$-resonance at $\sim 287.3 \mathrm{eV}[9,10]$.

The other XAS spectra also presented in Fig. 2 (spectra 2 and 3) show that irradiation of nano- and microsized diamonds by nitrogen ions with energy of $227 \mathrm{keV}$ and dose of $2.6 \cdot 10^{15}$ ions $/ \mathrm{cm}^{2}$ can produce just only moderate and reversible transformation of their bulk electronic structure. The noticeable decrease of $\mathrm{C} 1 \mathrm{~s}$ core exciton $(\sim 289.3 \mathrm{eV})$ gives evidence for the respective decrease of microdiamonds structural perfection. Some increase in the intensity of $\pi^{*}(\mathrm{C}=\mathrm{C})$ resonances $(\sim 285.1 \mathrm{eV})$ and corresponding decrease in the intensity of $\sigma^{*}$-resonances we regard as the formation of the surface layer of $\mathrm{sp}^{2}$-coordinated carbons. 


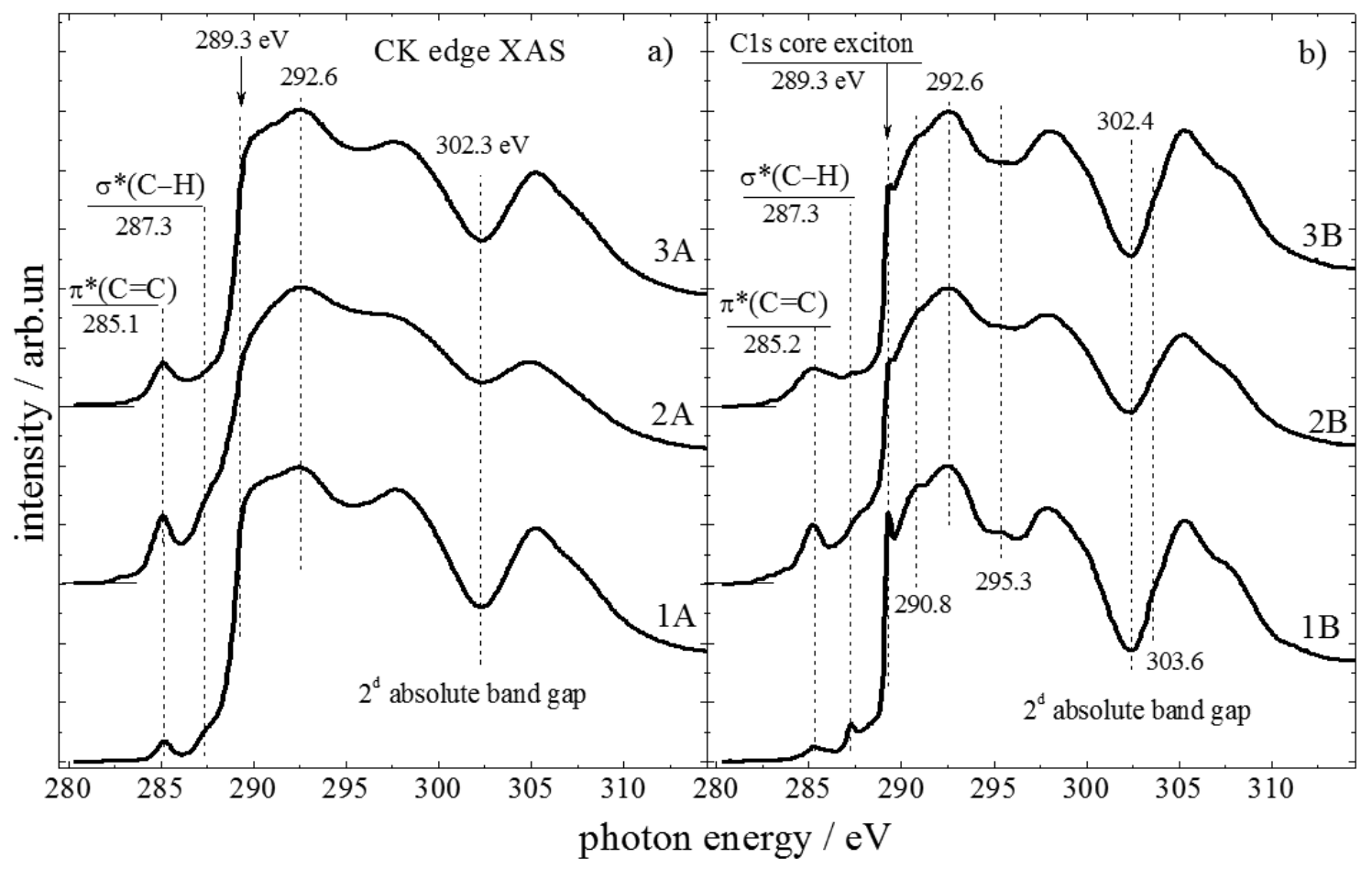

FIG. 2. CK edge XAS spectra of the samples prepared from deagglomerated DND (panel a) and synthesized diamond microcrystals (panel b). Spectra 1A, 1B correspond to the untreated diamond samples. Spectra 2A, 2B - to the same samples irradiated by nitrogen ions with energy of $227 \mathrm{keV}$ and dose of $2.6 \cdot 10^{15}$ ions $/ \mathrm{cm}^{2}$. Spectra $3 \mathrm{~A}, 3 \mathrm{~B}-$ the irradiated samples after their 1 -hour annealing in high vacuum conditions at $750{ }^{\circ} \mathrm{C}$

Comparison of spectra 2 and 3 shows that 1-hour annealing at $750{ }^{\circ} \mathrm{C}$ provides only partial restoration of the bulk structural perfection but inevitably results in an irreversible increase in the surface graphitization degree both for nano- and microdiamond particles.

Figure 3a presents typical PL spectra of the initial powder of DND (spectrum 1A) and of a high-quality type Ib single crystal diamond plate that was fabricated commercially by Element6 using HPHT growth and initially contained $200 \mathrm{ppm}$ of $N$. The sample was irradiated with $3-5 \mathrm{MeV}$ neutrons at the temperatures close to room temperature to a total dose of $10^{18} \mathrm{~cm}^{-2}$, then annealed at the temperature $T=800{ }^{\circ} \mathrm{C}$ for $2 \mathrm{~h}$ in the presence of a forming gas $\left(\mathrm{H}_{2}\right)$ in order to achieve a high density of $\mathrm{NV}^{-}$centers (Fig. 3a, spectrum 2A). The spectrum 1A featured by wide band of photoluminescence with maximum at wavelength $570 \mathrm{~mm}$ and the spectrum $2 \mathrm{~A}$ featured by wide band of photoluminescence with maximum at wavelength $680 \mathrm{~mm}$.

Figure $3 \mathrm{~b}$ (curve 1B) demonstrates PL spectrum of single crystal diamonds obtained by sintering of DND. It is featured by wide band of photoluminescence between 540 and $900 \mathrm{~nm}$. Bright band at the wavelength of $575 \mathrm{~nm}$ can be attributed to the emission of $\mathrm{N}-\mathrm{V}^{0}$ defects, points to significant concentration of them in diamond micro crystals sintered from DND.

Meanwhile, synthesized diamond microcrystals subjected to ion irradiation and subsequent annealing demonstrate the prominent transformation of the PL spectrum (Fig. 3b, curve 2B) approaching to the same of perfect single crystalline diamond (Fig. 3a, curve 2A). Maximum of PL spectrum of synthesized diamond microcrystals subjected to ion irradiation on subsequent annealing is as $680 \mathrm{~nm}$ compare to wide band of PL as $540 \mathrm{~nm}$ to $900 \mathrm{~nm}$ of non-irradiated synthesized diamond microcrystals.

The expressed line at $638 \mathrm{~nm}$ points to effective formation of luminescent $\mathrm{N}-\mathrm{V}^{-}$centers during processes of ionic irradiation and subsequent annealing.

\section{Conclusions}

Thus, HPHT sintering of $4-5 \mathrm{~nm}$ DND particles provides effective formation of $0.5-2 \mu \mathrm{m}$ sized single crystal diamonds with bulk electronic structure and structural perfection intrinsic to macroscopic high quality single crystal diamonds. Unlike initial DND, the single crystal diamonds obtained by sintering of DND contains 


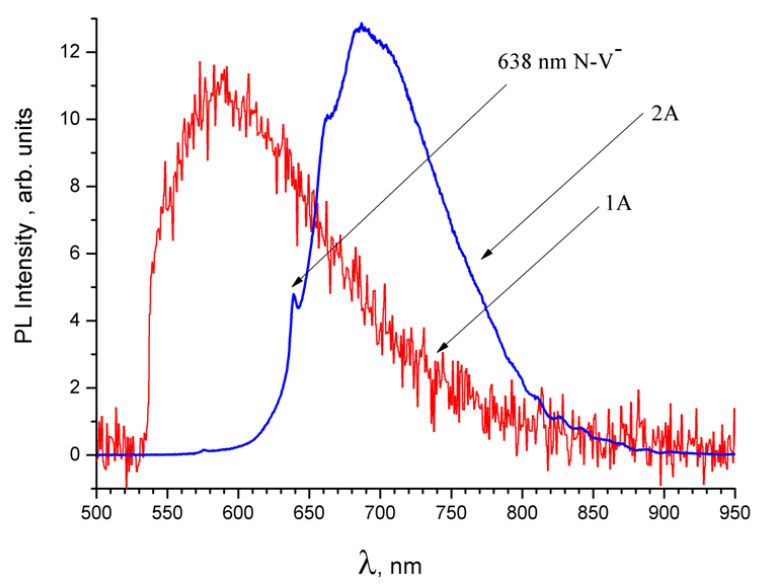

a

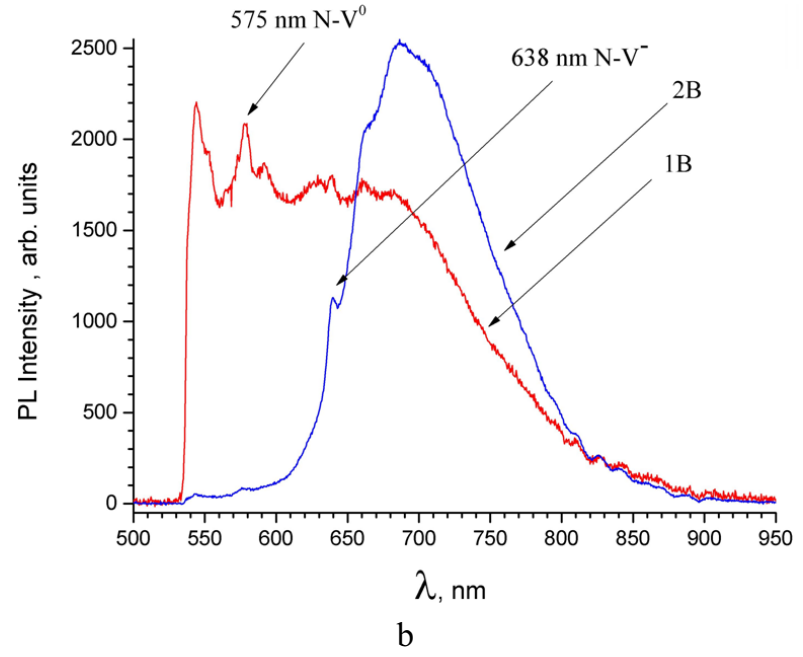

FIG. 3. a) Curve $1 \mathrm{~A}$ - Typical PL spectra of the initial powders of DND, curve 2A - PL spectra of high-quality synthetic diamond from Element6. b) curve 1B - typical PL spectrum of diamond microcrytals obtained by sintering of DND, curve $2 \mathrm{~B}-\mathrm{PL}$ spectrum of the same microdiamond after ion irradiation and annealing

no nitrogen in the near surface layers. Irradiation of synthesized microcrystalline diamond by $227 \mathrm{keV}$ nitrogen ions $\left(Q=2.51015\right.$ ions $\left./ \mathrm{cm}^{2}\right)$ and sequential 1-hour high vacuum annealing of these particles at the temperature of $750{ }^{\circ} \mathrm{C}$ results in the transformation of their PL spectra to the shape inherent to the perfect crystalline diamond with noticeable content of luminescent $\mathrm{N}-\mathrm{V}^{-}$centers. These results open the way to effective technologies of production the luminescent microdiamonds for various applications.

\section{Acknowledgements}

The S.V.K., A.Ya.V. acknowledge financial support of the Russian Science Foundation (project 14-13-00795). V.V.S., M.V.B., A.T.D., M.S.S. thank Russian German Laboratory at synchrotron BESSY II (Berlin) for the support of the XAS and XPS measurements and Helmholtz-Zentrum Berlin (HBZ) for the allocation of synchrotron radiation beam time. S.V.K. thanks V. Soltamov for providing the irradiated and annealed high-quality diamond (Element6) for testing.

\section{References}

[1] Kidalov S.V., Shakhov F.M., et al. Growth of diamond microcrystals by the oriented attachment. Tech. Phys. Lett., 2017,43 (1), P. 53-56.

[2] Dideikin A.T., Eidelman E.D., et al. Oriented-attachment growth of diamond single crystal from detonation nanodiamonds. Diam. Relat. Mater, 2017, 75, P. 85-90.

[3] Kidalov S.V., Shakhov, F.M., Vul' A.Ya., Ozerin A.N. Grain-boundary heat conductance in nanodiamond composites. Diam. Relat. Mater., 2010, 19 (7-9), P. 976-980.

[4] Osipov V.Yu., Shakhov F.M., et al. Identification of paramagnetic nitrogen centers (P1) in diamond crystallites synthesized via the sintering of detonation nanodiamonds at high pressure and temperature. Phys. Solid State, 2017, 59 (6), P. 1146-1153.

[5] Soltamova A.A., Ilyin I.V., et al. Detection and identification of nitrogen defects in nanodiamond as studied by EPR. Physica B: Condens. Matter, 2009, 404 (23-24), P. 4518-4521.

[6] Aleksenskiy A.E., Eydelman E.D., Vul' A.Ya. Deagglomeration of detonation nanodiamonds. Rehybridization of carbon on facets of detonation diamond nanocrystals and forming hydrosols of individual particles. Nanosci. Nanotech. Lett., 2011,3 (1), P. 68-74.

[7] Dideikin A.T., Aleksenskii A.E., et al. Phase transition on facets of detonation diamond nanocrystals and forming hydrosols of individual particles. Carbon, 2017, 122, P. 737-745.

[8] Molodtsov S.L., Fedoseenko S.I., et al. High-resolution RussianGerman beamline at BESSY. Appl. Phys. A, 2009 , 94, P. 501-505.

[9] Shpilman Z., Gouzman I., et al. A near edge X-ray absorption fine structure study of oxidized single crystal and polycrystalline diamond surfaces. Diam. Relat. Mater., 2014, 45, P. 20-27.

[10] Zagrebina E.M., Generalov A.V., et al. Comparative NEXAFS, NMR, and FTIR Study of Various-Sized Nanodiamonds: As-Prepared and Fluorinated. J. Phys. Chem. C, 2015, 119, P. 835-844. 